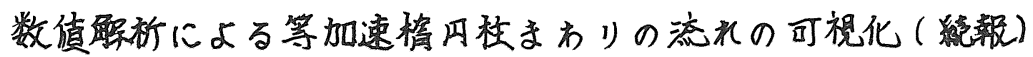

\author{
慶大理工 干河 晃・棚橋隆彦・安藤常世 \\ Flow visualization around a nuiformly accelerated elliptic cylinder \\ on a graphic display (PartII)
}

Akira CHINO* Takahiko TANAHASHI* Tsuneyo ANDO*

\begin{abstract}
In this paper, initial flows around an uniformly accelerated elliptic cylinder in an incompressible fluid are analysed from at rest by the finite difference method, and time histories of streamlines, equi-vorticity lines and drag coefficients are shown. The numerical schemes used here are the DuFort-Frankel technique for solving the vorticity equation and the Fourier series method for solving the stream function. For the latter case, the discrete Fourier transform and the Gauss elimination are applied to the analysis of a block tridiagonal matrix. Especially, with 70 average iterations per time step for the SOR method, the Fourier series method are verified to be more than 10 times faster.
\end{abstract}

\title{
1.まえがき
}

Navir-Stokes の方程式を基礎として、静止いている敤性・非区縮㟇流体中で静止

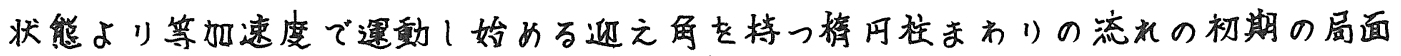
について、差分近似法を用いて解折いた。解析結果といて、時間経過とともに变 化す万流線、等浮度線物よだ抗力等の諸係数を求めたのでここに教告する。解析

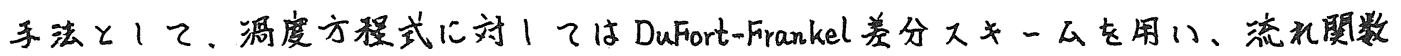

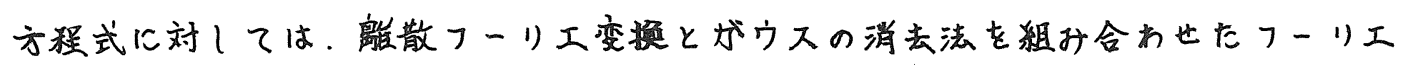
级数法を用いた。特に、後者の手法により大幅な訫算封間の短綰がはかられた。

\section{2. 解析方法}

\section{2-1主な記号}

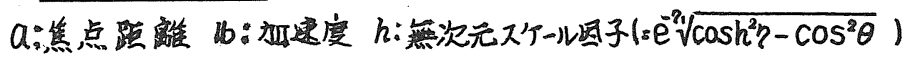

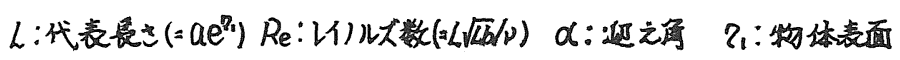

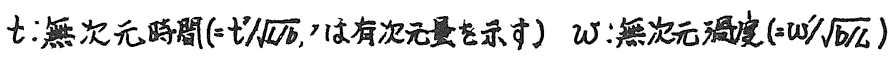

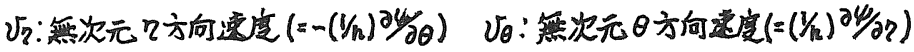

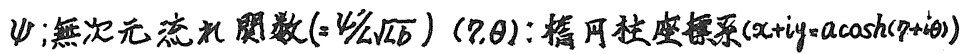

* Faculty of Science and Technology, Keio University, Yokohama.

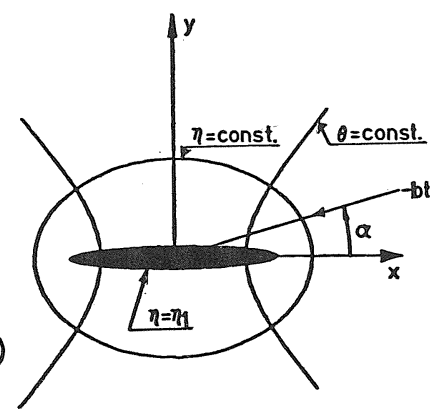

Fig.1 Model for analysis. 


\section{2-2。解析モデル}

Fig.1に示すような解析モデルを考える。すなわち、無限長さのスパンを特った

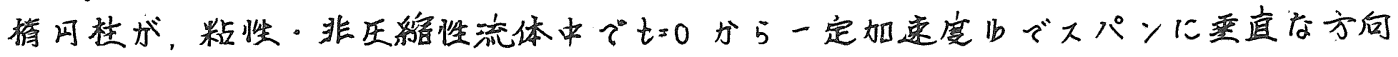

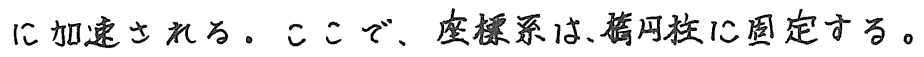

\section{2-3。基䃈方程式}

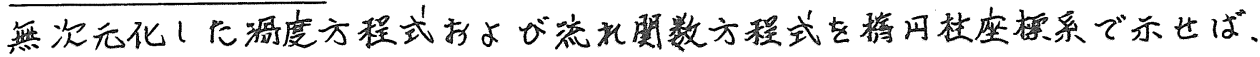

$$
\frac{\partial w}{\partial t}+\frac{1}{h^{2}} \frac{\partial(\psi, w)}{\partial(\eta, \theta)}=\frac{1}{R e} \nabla^{2} w \quad \text { (1) } \quad \nabla^{2} \psi=w
$$

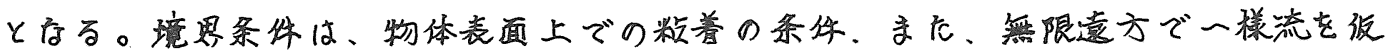
定てて次式を得る。

$$
\begin{aligned}
& \eta=\eta_{1}: \quad \psi=\partial \psi / \partial \eta=0 \\
& \eta \rightarrow \infty: \quad \partial \psi / \partial \theta=(1 / 2) e^{\eta-\eta_{1}}+\cos (\theta-\alpha) \quad, \quad \partial \psi / \partial \eta=(1 / 2) e^{\eta-\eta_{1}} t \sin (\theta-\alpha)
\end{aligned}
$$

また、物体表面に打流れの諸量より、物保の抗力・揚力・モーメン各係数が 算出でる。抗力係数CDは、CD

$$
\begin{aligned}
C_{D}= & \frac{2 e^{-\eta_{1}}}{R e}\left\{-\sinh \eta_{1} \cos \alpha \int_{0}^{2 \pi}\left(\frac{\partial w}{\partial \eta_{1}}\right)_{1} \sin \theta d \theta+\cosh \eta_{1} \sin \alpha \int_{0}^{2 \pi}\left(\frac{\partial w^{2}}{\partial \eta_{1}}\right) \cos \theta d \theta\right\}-2 \pi e^{-22_{1}} \sinh \eta_{1} \cosh \eta_{1} \\
& +\frac{2 e^{-\eta_{1}}}{R e}\left\{\cosh \eta_{1} \cos \alpha \int_{0}^{2 \pi} w_{1} \sin \theta d \theta-\sinh \eta_{1} \sin \alpha \int_{0}^{2 \pi} w_{1} \cos \theta d \theta\right\}
\end{aligned}
$$

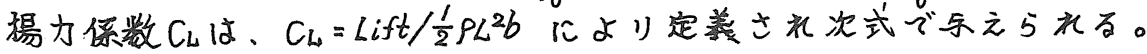

$$
\begin{aligned}
C_{L}= & \frac{2 e^{-\eta_{1}}}{R_{e}}\left\{\sinh \eta_{1} \sin \alpha \int_{0}^{2 \pi}\left(\frac{\partial w_{0}}{\partial \eta_{1}}\right) \sin \theta d \theta+\cosh \eta_{1} \cos \alpha \int_{0}^{2 \pi}\left(\frac{\partial w}{\partial \eta_{1}}\right) \cos \theta d \theta\right\} \\
& -\frac{2 e^{-\eta_{1}}}{R_{e}}\left\{\cosh \eta_{1} \sin \alpha \int_{0}^{2 \pi} w_{1} \sin \theta d \theta+\sinh \eta_{1} \cos \alpha \int_{0}^{2 \pi} w_{1} \cos \theta d \theta\right\}
\end{aligned}
$$

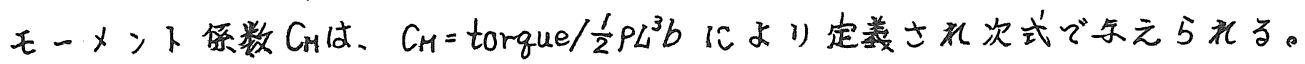

$$
C_{M}=\frac{e^{-2 \eta_{1}}}{R e} \int_{0}^{2 \pi}\left(\frac{\partial w}{\partial \eta}\right)_{1} \sin ^{2} \theta d \theta+\frac{2 e^{-2 \eta_{1}}}{R e} \sinh \eta_{1} \cosh \eta_{1} \int_{0}^{2 \pi} w_{1} d \theta
$$

$2-4$. 数值解析 ${ }^{(2)}$

基碳方程式を次のように韵散化し、差分近似により解析を行なう。

$$
\eta=\eta_{1}+\left(i^{\prime}-1\right) \Delta \eta \quad\left(i^{\prime}=1,2, \cdots, 4\right), \quad \theta=\left(j^{\prime}+0.5\right) \Delta \theta \quad\left(y^{\prime}=0,1,2, \cdots, M-1\right)
$$

滑度方程式(1)式に対しては、DuFort-Frankel法を用いて解析する。流れ閔方程 式(2)式に対】ては、フーリ工級数法を用いて解析する。才なわち、(2)式を中心差 分で近似才れば次のよう放无点公式を得る。

$$
2\left\{(\Delta \eta)^{2}+(\Delta \theta)^{2}\right\} \psi_{i, j}=(\Delta \theta)^{2}\left(\psi_{i v 1, j}+\psi_{i-1, j}\right)+(\Delta \eta)^{2}\left(\psi_{i, j+1}+\psi_{i, j-1}\right)-\left(\Delta \eta \cdot \Delta \theta h_{i, j}\right)^{2} \omega_{i, j}
$$

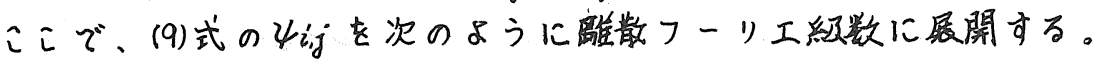

$$
\psi_{i, j^{\prime}}=\sum_{s=0}^{N}\left(a_{i, s}^{\prime} \cos \frac{\pi s}{N} j^{\prime}+b_{i, s} \sin \frac{\pi s}{N} j^{\prime}\right)
$$

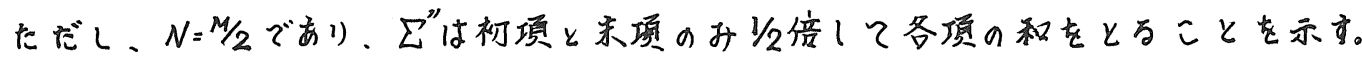

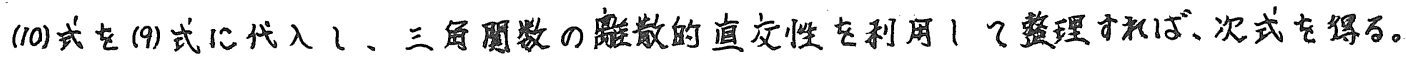




$$
\begin{aligned}
& -a_{i-1, s}^{\prime}+2\left\{\beta^{2}\left(1-\cos \frac{\pi}{N} s\right)+1\right\} a_{i, s}-a_{i+1, s}=-(\Delta \eta)^{2} \nabla_{i, s}^{i} \\
& -b_{i-1, s}+2\left\{\beta^{2}\left(1-\cos \frac{\pi}{N} s\right)+1\right\} b i, s-b i s 1, s=-(\Delta \eta)^{2} W_{i} s
\end{aligned}
$$

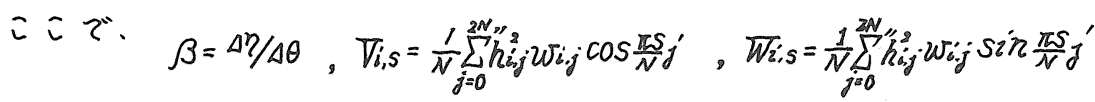

また、(3)(4)式の《に関する境界条件より次式を得る。

$$
\begin{aligned}
& a_{1,5}=0 \\
& b_{y, s}=0 \\
& 3 a_{L, S}-4 a_{L-1, S}+a_{L-2, S}=\Delta \eta S_{L, S} \quad . \quad 3 b_{L, S}-4 a_{L-1, S}+b l-2, S=\Delta \eta T_{L, S}
\end{aligned}
$$

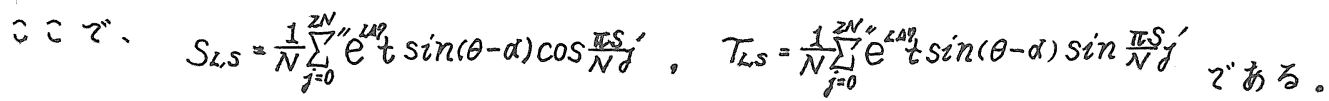

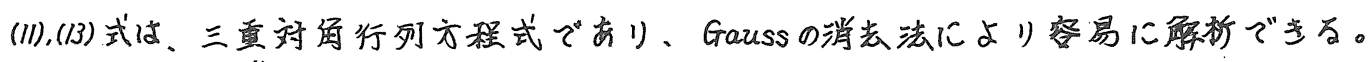
は执、Richtmyer (6) によれば $S=0$ 以外の時の氿旗差は小さくはる。

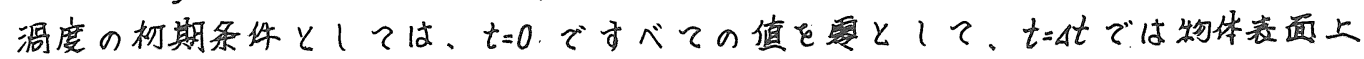

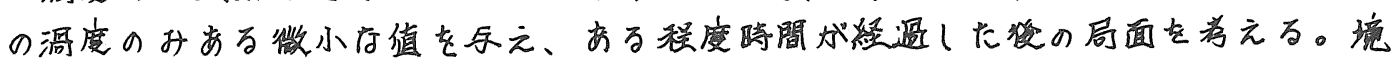
界条件としては次式を岳える。

$$
w_{l \cdot j^{\prime}}=0 \quad, w_{i \cdot j^{\prime}}=\left(\psi_{\text {i.j }}+44 \psi_{2 . j}-\psi_{3 . j^{\prime}}\right) /\left(2 k_{i j} \Delta \eta\right)^{2}
$$

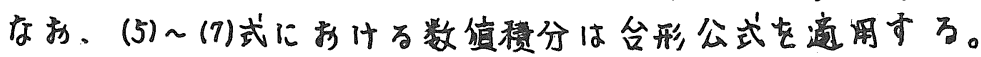
3. 解析结果

本解析に打ける内柱の場合 $\left(\eta_{1}=3.0\right)$ の流線と等渦度線の時間变化をFig.2 レイノルズ数は108.2である。また、Fig.3には、初期条件の影響を示1た。にれに

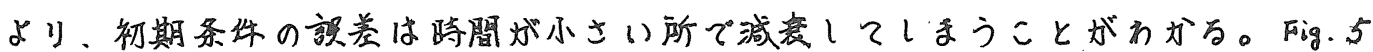

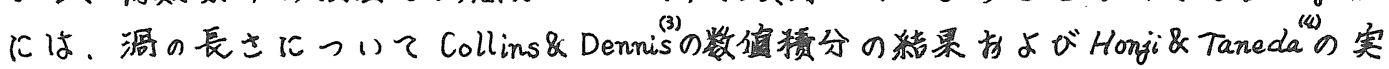

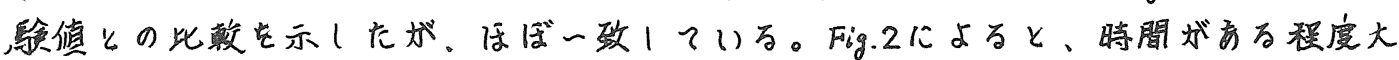

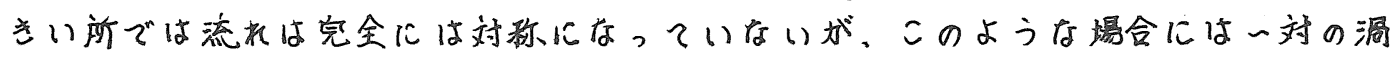

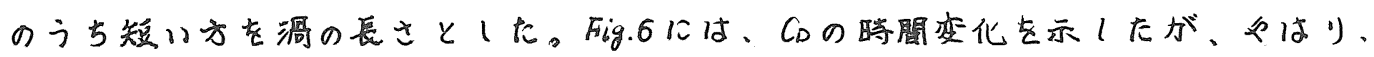

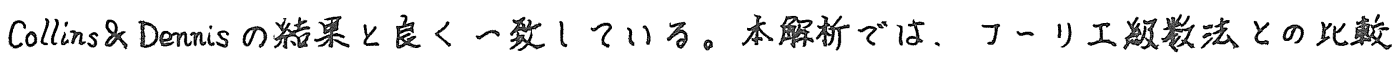
を行なうたりにSOR法による敢析吉行はった。Fig.6によると、前者は㣪首より

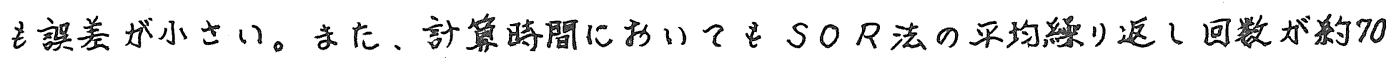
回である場合、フーリ工級数法は、SOR法むりも10倍以上速いことがわかった。

Fig.4では、4 $45^{\circ}$ の迎之苗を持った搰丹柱(

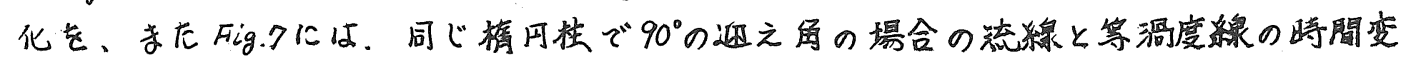
化を示けた。

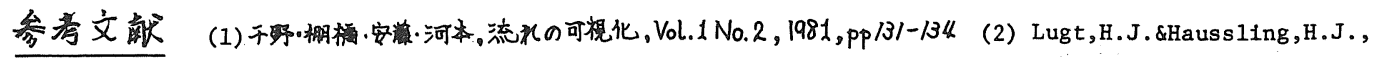
J.F1uid Mech.,65,1974,pp.711-734. (3) Collins,W.M.\&Denn1s, S.C.R., J.Fluid mech., 65, 1974,pp.461-480.

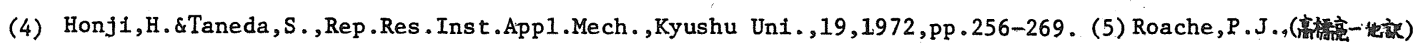

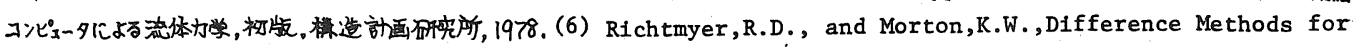
Initial-Value Problems, 2nd Edi., Interscience Publishers, J.Wiley and Sons, New York, 1967. (7). Taneda, S. and Honj1, H., J.Phys.Soc.Japan, 30(1971), pp262-272. 

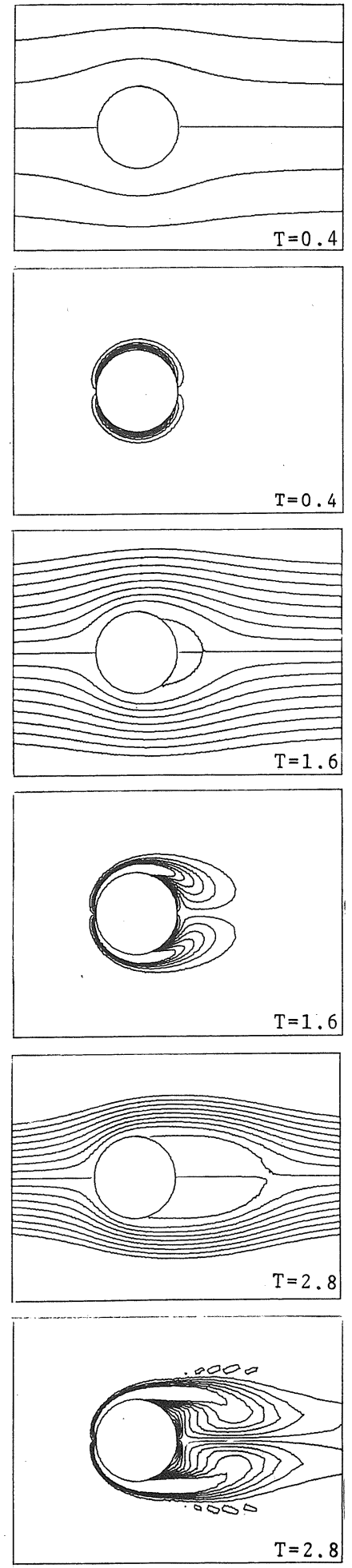
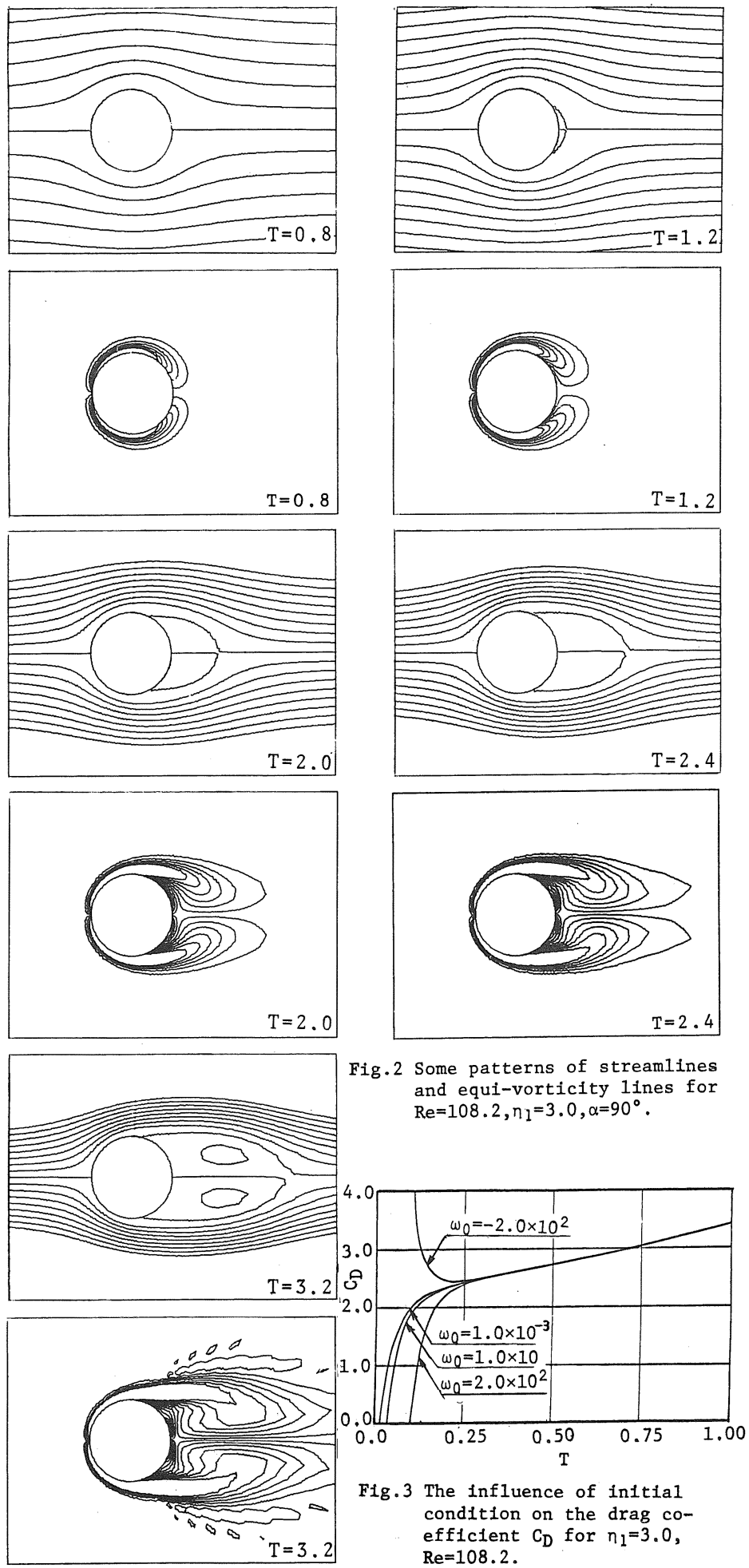

Fig. 3 The influence of initial condition on the drag coefficlent $C_{D}$ for $n_{1}=3.0$, $\operatorname{Re}=108.2$. 

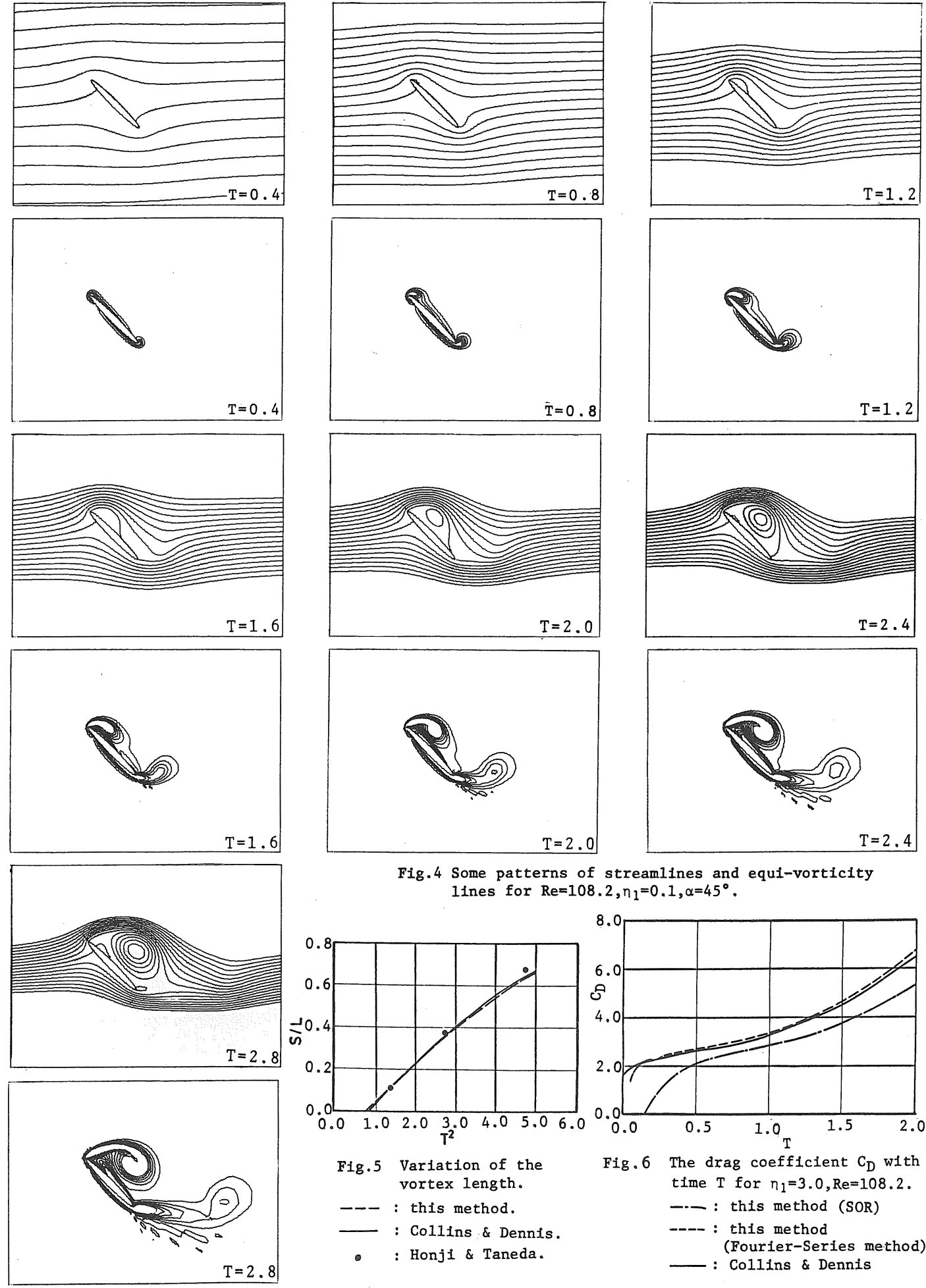

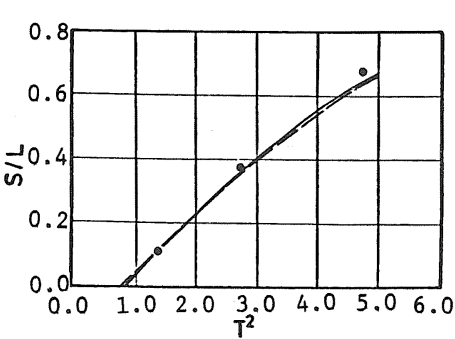

Fig.5 Variation of the vortex length.

- - : this method.

- : Collins \& Dennts.

- : Honji \& Taneda.

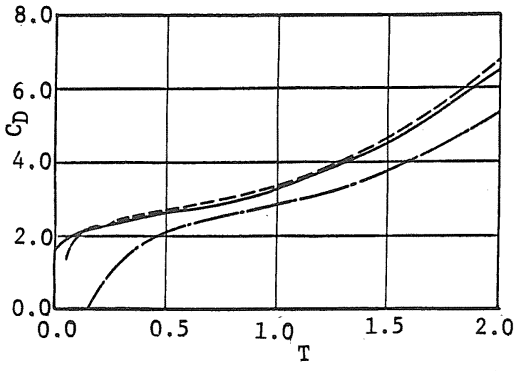

Fig.6 The drag coefficient $C_{D}$ with time $T$ for $n_{1}=3.0, R e=108.2$.

- - : this method (SOR)

-.-- : this method (Fourier-Series method) : Collins \& Dennis 

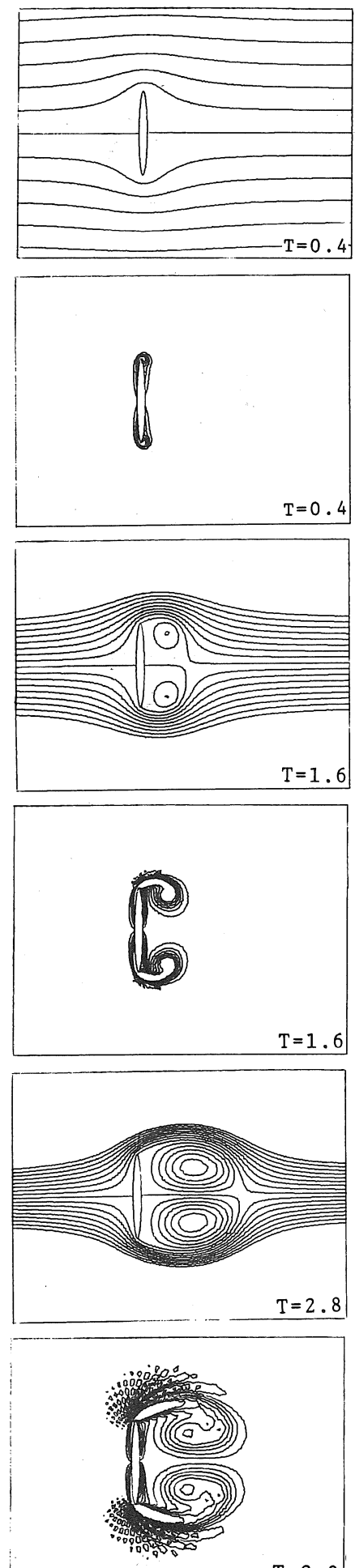

$\mathrm{T}=2.8$
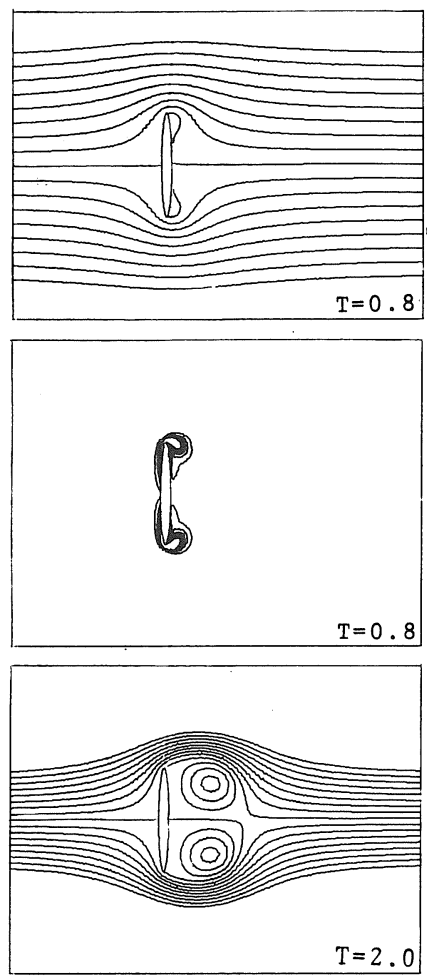

$\mathrm{T}=2.0$
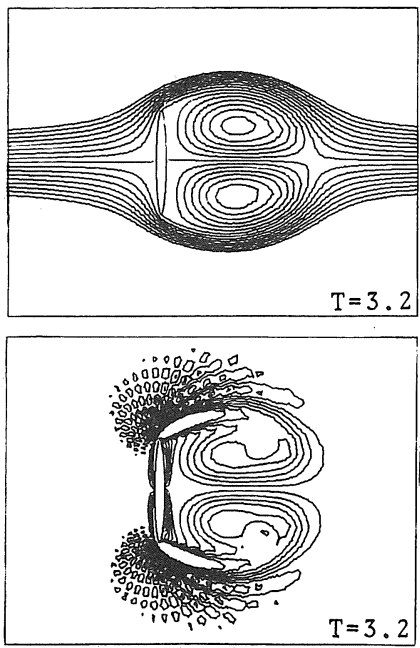

Fig. 8 The flow past an uniformly accelerated flat plate at (B) $\operatorname{Re}=98.1, t=2.9 \cdot($ Honj $1 \&$ Taneda)

Fig. 7 Some patterns of streamlines and equi-vorticity lines for $\operatorname{Re}=108.2, n_{1}=0.1, \alpha=90^{\circ}$.
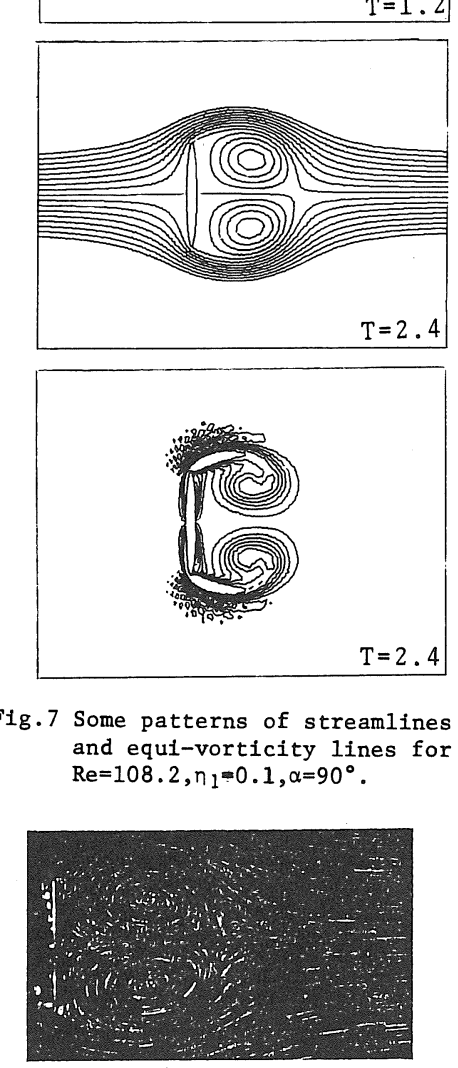